\title{
Acidic preconditioning reduces lipopolysaccharide-induced acute lung injury by upregulating the expression of angiotensin-converting enzyme 2
}

\author{
GUIYUAN YU ${ }^{1}$, YAN JIAO ${ }^{2}$, JIA-JIA HUANG ${ }^{3}$, MING-DA FAN $^{3}$, \\ $\mathrm{YU}_{-\mathrm{CHEN} \mathrm{HAO}}^{3}$, JI-ZHONG HAN ${ }^{4}$ and LIANGCHAO QU ${ }^{1,4}$ \\ ${ }^{1}$ Department of Anesthesiology, The First Affiliated Hospital of Nanchang University; \\ ${ }^{2}$ Department of Oncology, JiangXi Provincial People's Hospital, Donghu, Nanchang, Jiangxi 330000; \\ ${ }^{3}$ Medicine school of Nanchang University, Nanchang, Jiangxi 330006, P.R. China; ${ }^{4}$ Department of Physiology and \\ Biophysics, School of Medicine, Virginia Commonwealth University, Richmond, VA 23220, USA
}

Received June 28, 2020; Accepted January 15, 2021

DOI: $10.3892 / \mathrm{etm} .2021 .9879$

\begin{abstract}
Acid preconditioning (APC) through carbon dioxide inhalation can exert protective effects during acute lung injury (ALI) triggered by ischemia-reperfusion. Angiotensin-converting enzyme 2 (ACE2) has been identified as a receptor for severe acute respiratory syndrome coronavirus and the novel coronavirus disease-19. Downregulation of ACE2 plays an important role in the pathogenesis of severe lung failure after viral or bacterial infections. The aim of the present study was to examine the anti-inflammatory mechanism through which APC alleviates lipopolysaccharide (LPS)-induced ALI in vivo and in vitro. The present results demonstrated that LPS significantly downregulated the expression of ACE2, while APC significantly reduced LPS-induced ALI and provided beneficial effects. In addition, bioinformatics analysis indicated that microRNA (miR)-200c-3p directly targeted the 3'untranslated region of ACE2 and regulated the expression of ACE2 protein. LPS exposure inhibited the expression of ACE2 protein in A549 cells by upregulating the levels of miR-200c-3p. However, APC inhibited the upregulation of miR-200c-3p induced by LPS, as well as the downregulation of ACE2 protein, through the NF- $\mathrm{KB}$ pathway. In conclusion, although LPS can inhibit the expression of ACE2 by upregulating the levels of miR-200c-3p through the NF- $\kappa B$ pathway, APC inhibited this effect, thus reducing inflammation during LPS-induced ALI.
\end{abstract}

Correspondence to: Dr Liangchao Qu, Department of Anesthesiology, The First Affiliated Hospital of Nanchang University, 17 Yong Wai Zheng Road, Donghu, Nanchang, Jiangxi 330000, P. R. China

E-mail: liangchao.qu@outlook.com

Key words: acid preconditioning, acute lung injury, angiotensinconverting enzyme 2 , lipopolysaccharide

\section{Introduction}

Hypercapnia and hypercapnic acidosis have protective effects during lung injury induced by mechanical ventilation, ischemia-reperfusion, endotoxins and cecal ligations in numerous experimental models (1). In clinical treatment, hypercapnia has been linked to improvements in the outcomes of patients with acute lung injury (ALI)/acute respiratory distress syndrome (ARDS) $(2,3)$. Moreover, permissive or therapeutic hypercapnia also exerts protective effects during ALI, which involve the inhibition of key transcriptional activators in the NF- $\kappa \mathrm{B}$ pathway during inflammation (2-5). Our previous study confirmed that implementing acidic preconditioning (APC) by adjusting the respiratory frequency has a protective effect on ALI during the peri-operative period of mechanical ventilation in rat lung ischemia reperfusion (6). APC protocols are easy to operate and make the levels of hypercapnia controllable, thus increasing their applicability (6). Collectively, these aforementioned studies suggested that APC may represent a clinical application for the treatment of ALI. However, the mechanism of action through which APC reduces ALI remains poorly understood.

Angiotensin-converting enzyme (ACE) 2 exerts a protective effect on the cardiovascular system, liver and lungs (7-9). ACE2 inhibits the activity of ACE carboxypeptidase, which produces angiotensin II, the predominant active peptide of the renin-angiotensin system (RAS) $(10,11)$. ACE2 catalyzes the hydrolysis of angiotensin II and thus functions as a powerful negative regulator of the RAS, that balances the functions of ACE (12). Importantly, ACE2 has been identified as the receptor for severe acute respiratory syndrome coronavirus and the novel coronavirus disease-19 strain $(13,14)$. Moreover, the downregulation of ACE2 plays an important role in the pathogenesis of severe lung failure following viral infection (13-15).

Increasing evidence suggests that lipopolysaccharide (LPS) can induce ALI by downregulating ACE2 and upregulating angiotensin II $(16,17)$. Interestingly, the pathogenesis behind ALI/ARDS caused by viral and bacterial lung infection appears to share a similar mechanism involving reduced 
levels of ACE2 and increased levels of angiotensin II, which contribute to lung damage (18). Although these published studies established the relationship between LPS-induced ALI and ACE2, the role of APC on ACE2 in LPS-induced ALI remains unclear.

The aim of the present study was to examine the mechanisms of action through which APC alleviates LPS-induced ALI/ARDS. APC was implemented to both in vivo and in vitro models of lung injury induced by LPS. The expression of ACE2 and key inflammatory factors, as well as the severity of lung injury were measured. In addition, bioinformatics data suggested that microRNA (miRNA/miR)-200c-3p directly targeted the 3'unstranslated region (UTR) of ACE2. To test the hypothesis that miR-200c-3p regulates ACE2 expression in APC to alleviate LPS-induced ALI/ARDS, the effect of miR-200c-3p in LPS-induced lung injury following APC was evaluated in A549 cells. The functional role of miR-200c-3p on the regulation of ACE2 expression and the associated effects on LPS-induced lung injury was examined. As such, the present study identified a possible mechanism of action underlying the effects of APC on LPS-induced ALI.

\section{Materials and methods}

Model establishment and grouping. The animals were used with approval from the ethics committee of The First Affiliated Hospital of Nanchang University and all experiments undertaken in accordance with the relevant regulations. A total of 30 male Sprague-Dawley rats (Beijing Huafukang Bioscience Co., Ltd.), weighing 200-280 g and aged 8-10 weeks were placed in a professional animal breeding room, at a temperature of $18-26^{\circ} \mathrm{C}$ and relative humidity of $40-70 \%$, with a $12 \mathrm{~h}$ light/dark cycle and ad libitum access to food and water. The rats were anesthetized intraperitoneally with $50 \mathrm{mg} / \mathrm{kg}$ sodium pentobarbital and orally intubated with a sterile plastic catheter. The rats were randomly assigned into control, LPS and APC groups ( $n=10$ in each group). Rats in the control group were administered $50 \mu 1$ saline. In the LPS group, ALI was induced by intratracheal injections of $800 \mu \mathrm{g}$ LPS dissolved in $50 \mu \mathrm{l}$ saline (E. coli; model no. 055:B5; Sigma-Aldrich; Merck KGaA) (19). Rats in the APC group were given three 5 -min cycles of inhalation of mixed gas consisting of $20 \% \mathrm{CO}_{2}$ in normal air, followed by $10 \mathrm{~min}$ of recovery (20). After recovery, rats in this group also received $800 \mu \mathrm{g}$ LPS dissolved in $50 \mu 1$ saline. After the experiment, the rats were anesthetized intraperitoneally with $50 \mathrm{mg} / \mathrm{kg}$ pentobarbital sodium and the right atrium was opened to allow for the blood to flow until the heart stopped beating and death was confirmed.

Assessment of lung tissues. H\&E staining was performed to observe lung morphological injury. The right main bronchus was isolated and cross-clamped $12 \mathrm{~h}$ after LPS injection. Under constant pressure of $25 \mathrm{~cm} \mathrm{H}_{2} \mathrm{O}$, the left lung was filled with $0.5 \%$ low-melting agarose in $10 \%$ formalin through a tracheotomy to cause homogenous expansion of lung parenchyma. Subsequently, $10 \%$ formalin was used for fixation of the inflated lungs for $48 \mathrm{~h}$ in room temperature. The fixed samples were dehydrated by gradient concentrations of ethanol and paraffin blocks were used for embedding. H\&E (Thermo Fisher Scientific, Inc.) was used to stain the
5- $\mu \mathrm{m}$ paraffin-embedded tissue sections. The sections were stained in room temperature as follows: $70 \%$ ethanol for $10 \mathrm{sec}$, diethyl pyrocarbonate-treated water for $5 \mathrm{sec}$ and hematoxylin with RNAase inhibitor for $20 \mathrm{sec}$. Subsequently, $70 \%$ ethanol was applied for $30 \mathrm{sec}$, eosin Y in 100\% ethanol for $20 \mathrm{sec}$, followed by dehydration with a series of ethanol for $30 \mathrm{sec}$ each, and finally xylene for $2 \mathrm{~min}$. Histopathological changes in lung tissues were observed under light microscope (Olympus Corporation; magnification, x100 and x400). The inflammatory cell infiltration was evaluated as a count of the average number of neutrophils in 5 high power fields. The lung injury score was calculated by hyaline membranes formatting, alveolar wall thickening and airspaces filling with proteinaceous debris, as previously described (6). Each item is scored from zero to four, of which zero points represent normal, one point represents a very small change, two points represent a slight change, three points represents moderate change and four points represent a serious change. The pathological score of lung tissue was the average of each score. The wet/dry (W/D) lung tissue weight ratio was used to assess the degree of lung edema (6).

Immunofluorescence microscopy For ACE2 immunofluorescence assessment, a polyclonal rabbit anti-ACE2 antibody (1:1,000; Abcam; cat. no. ab108252) was used. Frozen lung tissues were sectioned into $6 \mu \mathrm{m}$-thick slices, permeabilized ( $0.25 \%$ Triton X-100), quenched and blocked (10\% goat serum) for $1 \mathrm{~h}$ at room temperature, after which samples were incubated with the polyclonal rabbit anti-ACE2 antibody (1:100 diluted in PBS) overnight at room temperature. Primary antibody-bound ACE2 was subsequently visualized using fluorescein isothiocyanate-conjugated anti-rabbit IgG antibodies (1:1,000; Abcam; cat. no. R330217) for $2 \mathrm{~h}$ at room temperature. The distribution of the target protein in cells was analyzed by fluorescence microscopy (Leica Microsystems, Inc.; magnification, x400).

Potential miRNAs that target ACE2 in ALI. To predict the potential miRNAs that regulate the expression of the ACE2 protein, TargetScan (Release 7.2: March 2018; http://www. targetscan.org/vert_72/) was used to search for predicted miRNA targets in the human genome. In addition, the miR binding site on the ACE2 3'-UTR was identified using TargetScan (http://www.targetscan.org/cgi-bin/targetscan/ vert_72/view_gene.cgi?rs=ENST00000252519.3\&taxid=9606 $\&$ showcnc $=0 \&$ shownc $=0 \&$ shownc $\_n c=\&$ showncf $1=\&$ shown cf $2=\&$ subset=1). ACE2 serves an important role in virus-induced lung injury, which is similar to LPS-induced lung injury (21). The reported top 20 highly expressed miRNAs in H5N1 virus-infected A549 cells (18) were selected to predict the potential miRNAs that target ACE2 in LPS-induced lung injury.

Cell culture. The human A549 lung adenocarcinoma epithelial cell line was obtained from the American Type Culture Collection and cultured in $5 \% \mathrm{CO}_{2}$ at $37^{\circ} \mathrm{C}$ in Ham's F12 nutrient medium (HyClone; Cytiva) supplemented with $10 \%$ of FBS (Hyclone; Cytiva), $100 \mu \mathrm{g} / \mathrm{ml}$ of streptomycin and $100 \mathrm{IU} / \mathrm{ml}$ of penicillin. For LPS stimulation, A549 cells were harvested, seeded (500 cells/well) in 6-well plates and incubated overnight at $37 \mathrm{C}$. Samples were then treated with $1 \mu \mathrm{g} / \mathrm{ml}$ LPS for $24 \mathrm{~h}$. To 
replicate APC conditions, cells were exposed to $20 \% \mathrm{CO}_{2}$ for $4 \mathrm{~h}$ before receiving LPS $1 \mu \mathrm{g} / \mathrm{ml}$. The specific inhibitor of NF- $\kappa \mathrm{B}$, caffeic acid phenethyl ester (CAPE; TargetMol) $75 \mu \mathrm{mol} / 1$, was added to cells for $2 \mathrm{~h}$ before LPS exposure. The native group was cultured without LPS, the control group was cultured with $1 \mu \mathrm{g} / \mathrm{ml}$ LPS for $24 \mathrm{~h}$ and the CAPE group was cultured with CAPE $75 \mu \mathrm{mol} / 1$ for $2 \mathrm{~h}$, after which $1 \mu \mathrm{g} / \mathrm{ml}$ LPS was applied for $24 \mathrm{~h}$. The APC group was exposed to $20 \% \mathrm{CO}_{2}$ for $4 \mathrm{~h}$, after which $1 \mu \mathrm{g} / \mathrm{ml}$ LPS was applied for $24 \mathrm{~h}$. All treatment and culture incubations were performed at $37^{\circ} \mathrm{C}$.

Cell transfection. A549 cells were transfected with miR-200c-3p mimics or miR mimics control $50 \mathrm{nmol} / 1$ (Shanghai GenePharma Co.,Ltd.) at $37^{\circ} \mathrm{C}$ for $36 \mathrm{~h}$. Transfections were performed using Lipofectamine RNAiMAX (Invitrogen; Thermo Fisher Scientific, Inc.) according to the manufacturer's instructions. The sequences were as follows: miR-200c-3p mimics, 5'-UAAUACUGCCGGGUAAUGAUGGA-3'; miR mimics control, 5'-UUGUACUACACAAAAGUACUG-3'.

Western blot analysis. After transfections, the A549 cells, the cells were treated as aforementioned (control, LPS and APC groups) and collected in RIPA lysis buffer (Beijing BLKW Biotechnology Co., Ltd.). Protein expression levels were quantified using BCA Protein Assay kit (Thermo Fisher Scientific, Inc.). Total protein $(40 \mathrm{mg}$ ) was loaded onto $10 \%$ SDS-PAGE gels. After electrophoresis, the proteins were transferred to a PVDF supporting membrane. The membrane was incubated for $12 \mathrm{~h}$ at $4^{\circ} \mathrm{C}$ with primary antibodies targeting ACE2 (1:1,000; Abcam; cat. no. ab108252) or $\beta$-actin (1:1,000; Sigma-Aldrich; Merck KGaA). Following 15 min washes with TBS-0.1\% Tween-20. The membranes were blocked with $5 \%$ non-fat milk at room temperature for $1 \mathrm{~h}$ and incubated with HRP-conjugated secondary antibodies (1:10,000; Cell Signaling Technology, Inc.; cat. no. 7074) for $1 \mathrm{~h}$ at room temperature. Blots were developed using an ECL kit (cat. no. P0018; Beyotime Institute of Biotechnology). The protein expression was subsequently analyzed using ImageJ software 1.46 (National Institutes of Health).

Reverse transcription-quantitative PCR (RT-qPCR) analysis. Total RNA was isolated from the cultured cells using the Eaststep Total RNA Extraction kit (Promega Corporation) according to the manufacturer's instructions. Subsequently, RNA was reverse transcribed using a Transcriptor First-Stand cDNA Synthesis kit (TransGen Biotech Co., Ltd.) according to the manufacturer's protocol. qPCR was performed using the TransStart Green qPCR SuperMix (TransGen Biotech Co., Ltd.) on a DA7600 real-time nucleic acid amplification fluorescence detection system (Bio-Rad Laboratories, Inc.). The thermocycling conditions were as follows: $98^{\circ} \mathrm{C}$ for $15 \mathrm{~min}$, and $96^{\circ} \mathrm{C}$ for $5 \mathrm{~min}$ (initiation), $92^{\circ} \mathrm{C}$ for $30 \mathrm{sec}$ (denaturation), $65^{\circ} \mathrm{C}$ for $30 \mathrm{sec}$ (annealing) and $62^{\circ} \mathrm{C}$ for $1 \mathrm{~min}$ (extension) for 40 cycles. The transcript of the housekeeping gene GAPDH was quantified as a reference gene. All primers were designed and optimized using Oligo 7.0 software (Molecular Biology Insights) and synthesized by Sangon Biotech. The following primers were used for amplification: i) ACE2 forward, 5'-CATTGGAGCAAGTGT TGGATCTT-3'; and reverse, 5'-GAGCTAATGCATGCC
ATTCTC-3'; ii) IL-1 $\beta$ forward, 5'-TGGACCTTCC-AGGAT GAGGACA-3'; and reverse, 5'-GTTCATCTCGGAGCCTGT AGTG-3'; iii) IL-6 forward, 5'-AGTGAGGAACAAGCCAGA GC-3'; and reverse, 5'-CAGGGGTGGTTATTGCATCT-3'; and iv) GADPH forward, 5'-AGGGCTGCTTTTAACTCT GGT-3'; and reverse, 5'-CCCCACTTGAT-TTTGGAGGGA-3'. The primers for miR-200c-3p (forward, 5'-AGGGCTAAT ACTGCCGGGTAA-3'; reverse, 5'-GTCGTATCCAGTGCA GGGTCCGAGGTATTCGCACTGGATACGACTCCATC-3') were purchased from GenoExplorerTM (GenoSensor Corporation). The U6 small nuclear ribonucleoprotein primer (forward, 5'-CGCTTCGGCAGCACATATAC-3'; reverse, 5'-TTCACGAATTTGCGTGTCAT-3') was used to normalize miRNA levels. The target gene were standardized to the $\mathrm{Cq}$ values of GAPDH $(\triangle \mathrm{Cq})$ to calculate the relative protein expression levels of the target genes, and the value was determined as $2^{-\Delta \Delta \mathrm{Cq}}(22)$.

Statistical analysis. Data are presented as the mean \pm SD. Statistical analysis was carried out using unpaired Student's t-test. One-way ANOVAs were used to evaluate the effect and pathological characteristics of chemotherapy. Tukey's tests were used for the post hoc tests to determine significant differences between groups following a significant ANOVA result. Data that was not continuous were analyzed using Kruskal-Wallis tests and Dunn's tests for the post hoc tests. $\mathrm{P}<0.05$ was considered to indicate a statistically significant difference.

\section{Results}

$A P C$ reduces acute lung injury induced by $L P S$. To identify the effects of APC on LPC-induced acute lung injury, LPS was administered to rats by intratracheal injection. The lung tissue was obtained $12 \mathrm{~h}$ after injection. Compared with the control group, LPS induced massive neutrophil infiltration in alveoli and focal alveolar wall thickenings. In addition, compared with the LPS group, APC reduced inflammatory exudation (Fig. 1A). In addition, the APC group displayed reduced inflammatory cell infiltration compared with the LPS group (Fig. 1B). The lung injury score also decreased in the APC group (Fig. 1C). Compared with the LPS group $(6.81 \pm 1.37)$, APC (5.62 \pm 1.72$)$ significantly improved the W/D tissue weight ratio in LPC-induced ALI (Fig. 1D).

$A P C$ inhibits inflammation by reversing $L P S$-induced downregulation of ACE2 expression. ACE2 plays an important role in LPS-induced lung injury (23). In order to determine the effect of ACE2 protein expression on APC inhibition of LPS-induced ALI, immunofluorescence staining was used to examine the expression of ACE2 protein in A549 cells (Fig. 2A). Fluorescence intensity results demonstrated that, compared with the control group, ACE2 expression in the LPS group $(0.63 \pm 0.11)$ was significantly reduced, while APC $(0.81 \pm 0.17)$ reversed the LPS-induced downregulation of ACE2 expression (Fig. 2B). In addition, compared with the control group, LPS significantly reduced the expression levels of ACE2 (Fig. 2C), while IL-1 $\beta$ and IL-6 mRNA expression levels significantly increased (Fig. 2D and E). The protein expression levels of ACE2 increased in the APC group, while 

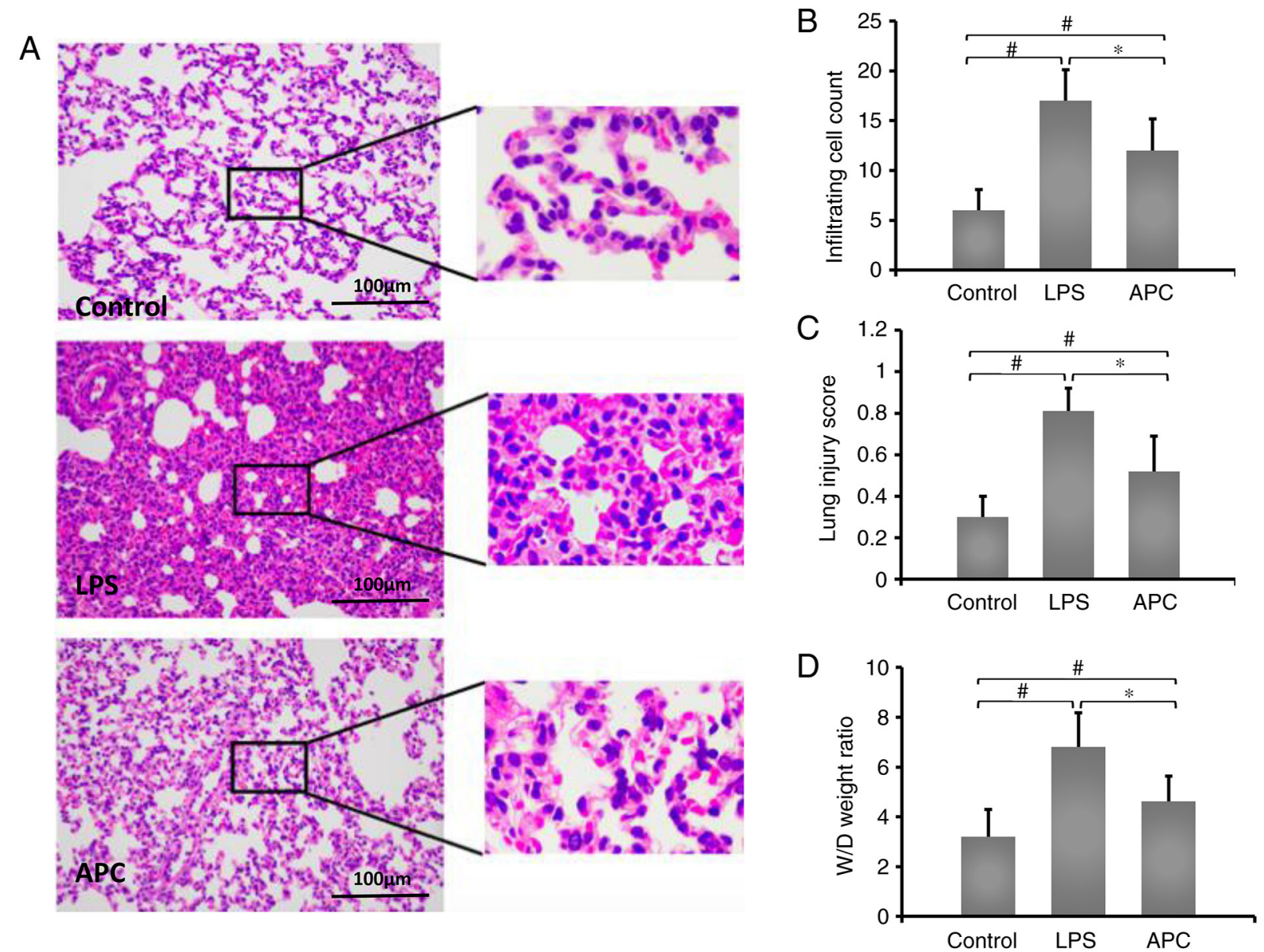

Figure 1. Effect of APC on LPS-induced acute lung injury. (A) Representative images of lung pathology (H\&E) of LPS-induced rats treated with APC. (B) Number of infiltrating neutrophils per microscopic field. A total of 50 fields were analyzed. $n=10$ in each group. (C) Lung injury scores including alveolar wall thickening, hyaline membranes formatting and airspaces filling with proteinaceous debris in different groups. (D) W/D lung tissue weight ratios indicating the degree of lung edema were detected $12 \mathrm{~h}$ after LPS induction. Data are presented as the mean $\pm \mathrm{SD} . \mathrm{n}=10$. ${ }^{*} \mathrm{P}<0.05$ and ${ }^{\#} \mathrm{P}<0.01$. APC, acid preconditioning; LPS, lipopolysaccharide; W/D, wet/dry.

the mRNA expression levels of IL-1 $\beta$ and IL- 6 decreased significantly, compared with the LPS group. These results demonstrated that APC played a crucial protective role in regulating the expression of ACE2 and inhibiting LPS-induced ALI.

Potential miRNAs that target ACE2 in ALI. Using TargetScan online tool, 281 miRNAs were identified that may regulate ACE2 protein, which contained 3 conserved sites and 278 poorly conserved sites (Table S1). The top 20 expressed miRNAs were chosen for the subsequent predictions. The reported top 20 highly expressed miRNAs in H5N1 virus-infected A549 cells were selected (18) and the $\log _{2}$ fold changes of miRNA expression were listed (Fig. 3A). The intersection between the top 20 highly expressed miRNAs in virus-infected A549 cells and the top 20 miRNAs regulating the expression of ACE2 protein was identified (Fig. 3C). Only one miRNA matched this condition, namely miR-200c-3p, which was partially similar to the miRNAs identified in a previous report (18). miR-200c-3p and miR-141-3p may have effects on the expression of ACE2 protein during $\mathrm{H} 5 \mathrm{~N} 1$ virus infection. The miR-200c-3p binding site on the ACE2 3'-UTR was found and is displayed in Fig. 3C.
Effects of APC on the reduction of LPS-induced ALI by $m i R-200 c-3 p / A C E 2$. To confirm the bioinformatics analysis, the relative expression levels of miR-200c-3p and ACE2 were measured using RT-qPCR (Fig. 4A and B). APC did not demonstrate a marked effect on normal A549 cells. Following exposure to LPS, the relative expression levels of miR-200c-3p increased significantly in A549 cells, while the relative expression of ACE2 decreased significantly. Compared with the control LPS treated group, APC downregulated the expression of miR-200c-3p and increased the expression of ACE2 in LPS-treated A549 cells. To demonstrate the interaction between APC and miR-200c-3p in LPS-induced lung injury, the miR-200c-3p mimics were successful transfected into A549 cells. It was found that the relative expression of miR-200c-3p increased compared to the miR mimic controlled transfected cells (Fig. 4C), indicating that the transfection was successful. In addition, following miR-200c-3p mimic transfection in A549 cells, the protein expression levels of ACE2 were significantly downregulated (Fig. 4D), and compared with the native group, APC increased the expression of ACE2 in LPS-treated A549 cells. This effect was reversed by miR-200c-3p mimic transfection (Fig. 4E).

Previous studies have demonstrated that NF- $\mathrm{KB}$ plays a role in the regulation of miR-21, miR-125b-1, miR-21, miR-30b, 

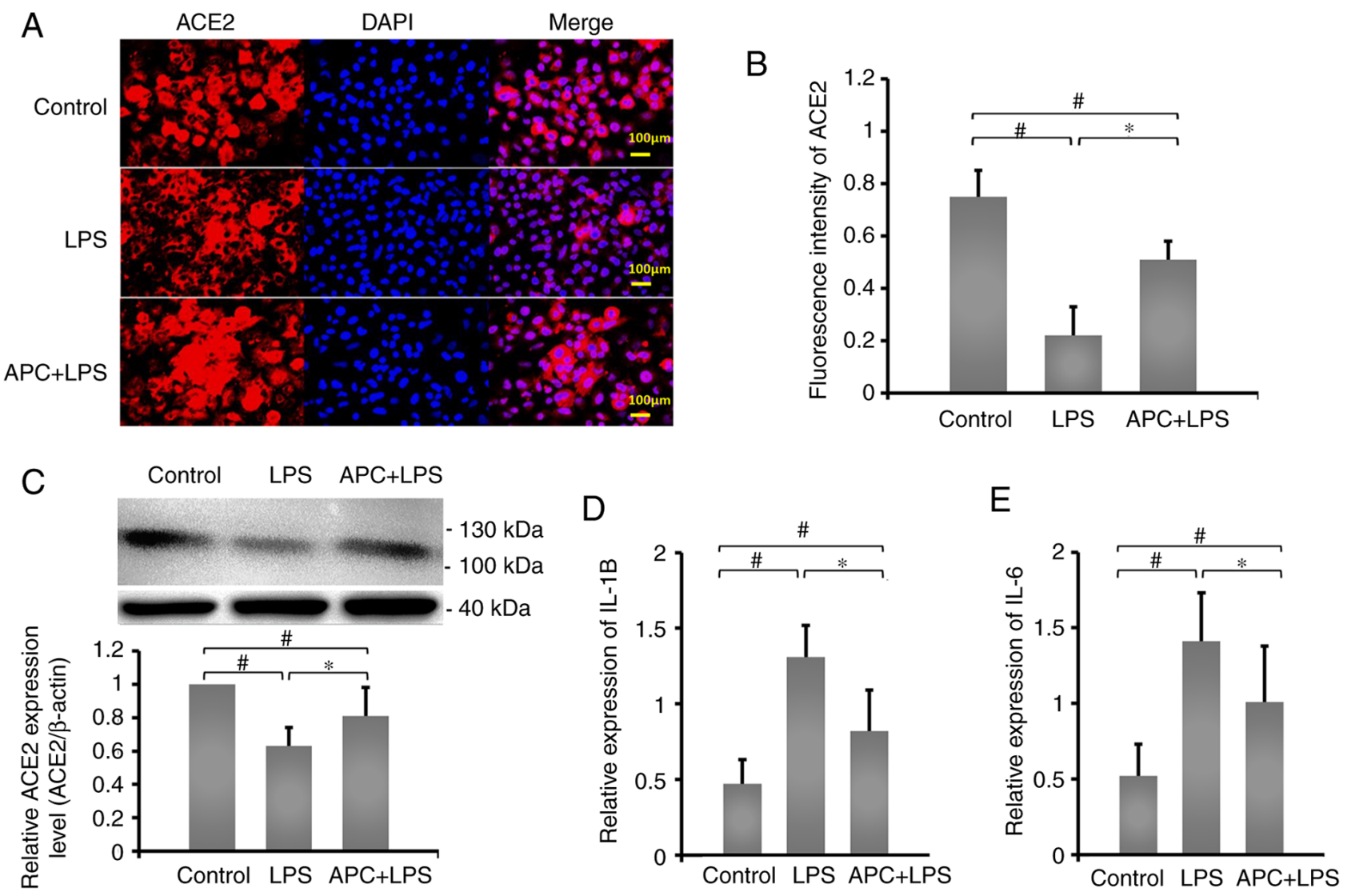

Figure 2. APC inhibits inflammation in LPS-treated A549 cells by upregulating ACE2 expression. (A) Representative immunofluorescence staining images of ACE2 expression in the various groups of A549 cells. Red fluorescence shows ACE2, the blue fluorescence shows the nucleus following DAPI staining.

(B) ACE2 fluorescence intensity results in each group. (C) Expression of ACE2 in A549 cells following 24-h challenge with $1 \mu \mathrm{g} / \mathrm{ml} \mathrm{LPS}$. (D) IL-1 $\beta$ and (E) IL-6 mRNA levels in A549 cells following 24-h treatment with $1 \mu \mathrm{g} / \mathrm{ml}$ LPS. Data are presented as the mean \pm SD. $\mathrm{n}=3$. "P<0.05 and "P<0.01. ACE2, angiotensin-converting enzyme 2; LPS, lipopolysaccharide.

\section{A Top 20 highily expressed miRNAs}

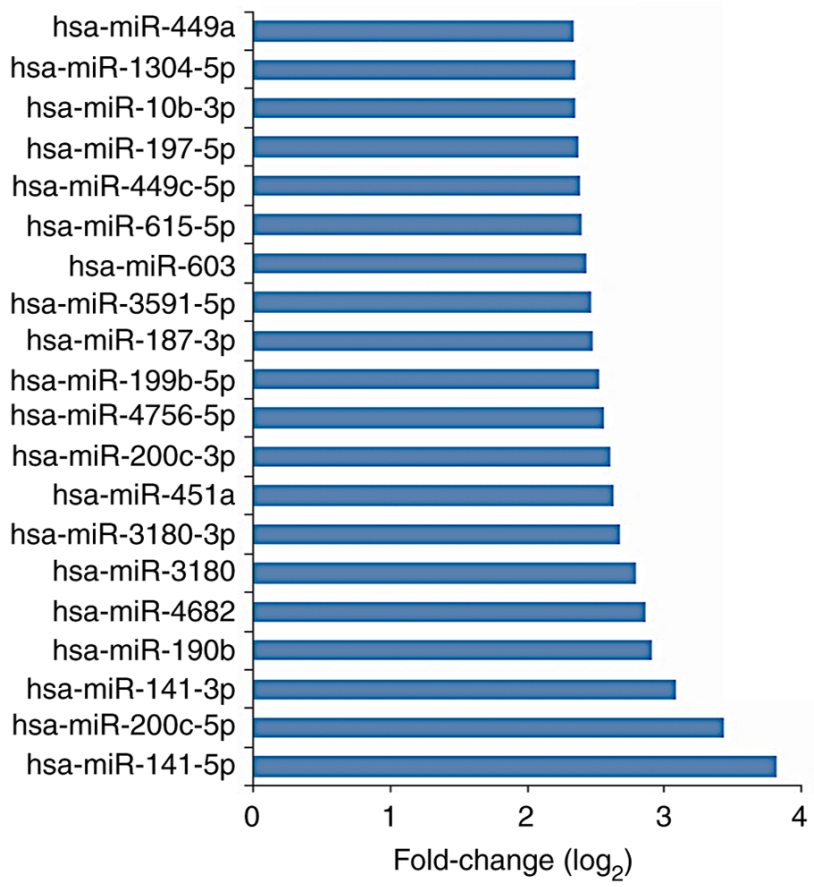

B

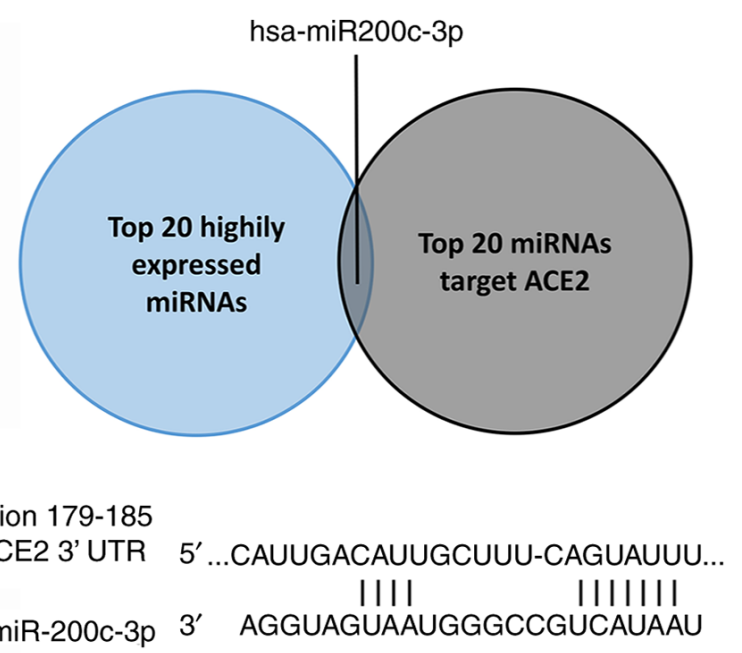

Figure 3. Potential miRNAs regulating the protein expression levels of ACE2. (A) Top 20 highly expressed miRNAs in H5N1 virus-infected A549 cells. (B) Intersection between the top 20 highly expressed miRNAs in the virus-infected A549 cells and the top 20 miRNAs regulating the expression of ACE2 protein. (C) The miR-200c-3p binding site on the ACE2 3'-UTR. ACE2, angiotensin-converting enzyme 2; miR/miRNA, microRNA; UTR untranslated region. 


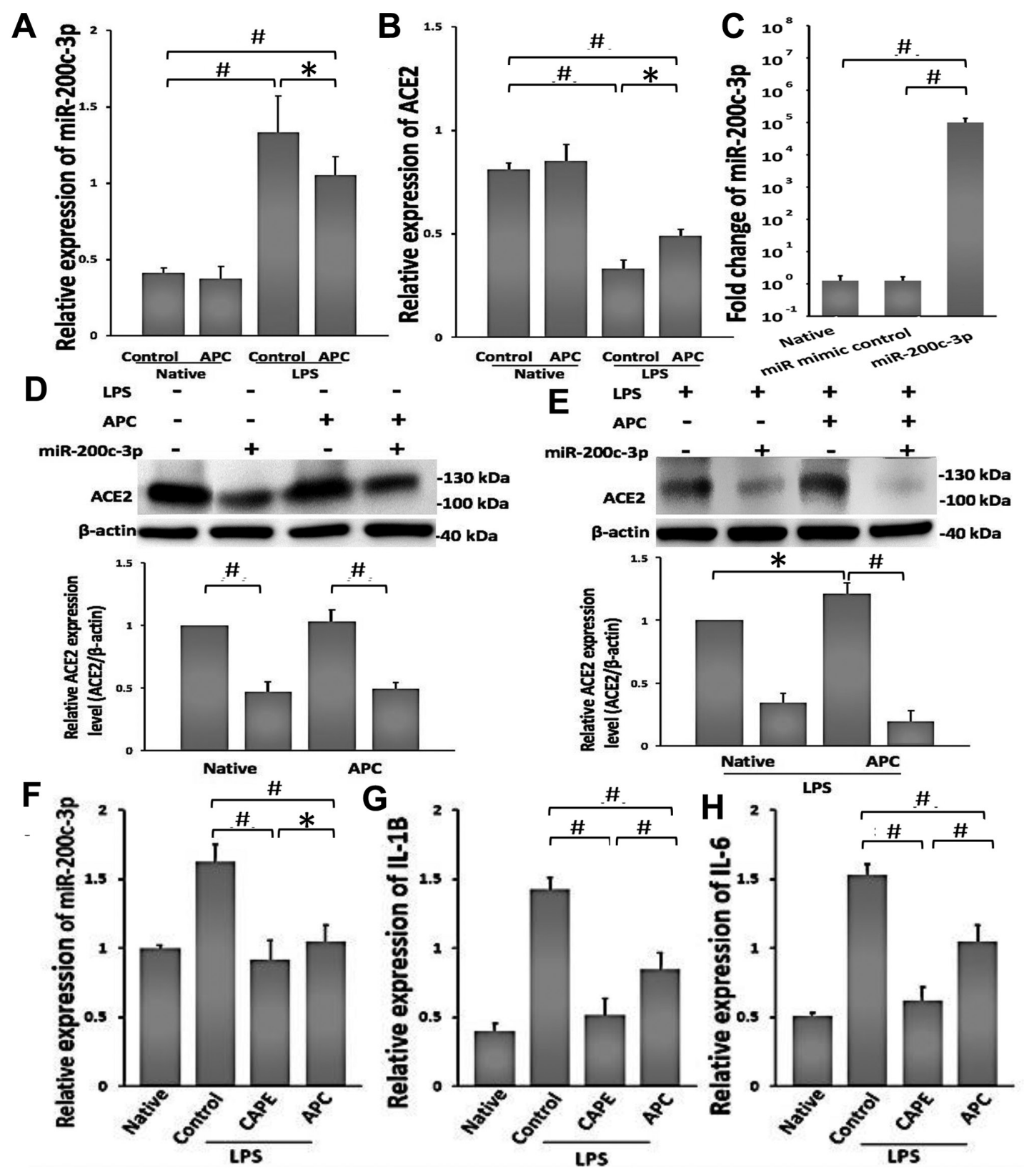

Figure 4. APC protects against LPS-induced lung injury by downregulating miR-200c-3p and increasing ACE2 expression. (A and B) RT-qPCR analysis of the expression levels of miR-200c-3p and ACE2 in A549 cells following 24-h treatment with $1 \mu \mathrm{g} / \mathrm{ml}$ LPS. Native, untreated with LPS in A549 cells. (C) The transfection efficiency of miR-200c-3p mimics was detected using RT-qPCR. A549 cells were transfected with mimics of miR-200c-3p for $36 \mathrm{~h}$. ACE2 protein expression levels were analyzed following further treatment (D) without LPS or (E) with LPS, using western blotting and were normalized to $\beta$-actin. Native

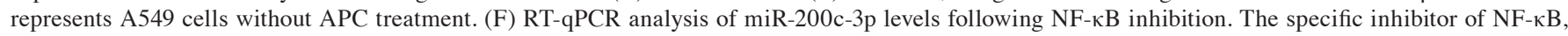
CAPE, was added to cells for $2 \mathrm{~h}$ before LPS exposure. Native represents cells without LPS treatment. RT-qPCR analysis of the expression of (G) IL-1 $\beta$ and (H) IL-6 in A549 cells after 2-h treatment with CAPE. Native represents cells without LPS treatment. Data are presented as the mean \pm SD. $n=3$. ${ }^{*}<<0.05$ and ${ }^{\text {"P}} \mathrm{P}<0.01$. ACE2, angiotensin-converting enzyme 2; APC, acid preconditioning; CAPE, caffeic acid phenethyl ester; LPS, lipopolysaccharide; miR, microRNA; RT-qPCR, reverse transcription-quantitative PCR.

miR-23b-27b-24-1 and miR-200c-3p expression (18,24,25). As such, CAPE significantly reduced the levels of miR-200c-3p, compared with LPS-exposed cells. In addition, APC also resulted in the inhibition of the upregulation of miR-200c-3p induced by LPS (Fig.4F). The expression levels of inflammatory factors (IL-1 $\beta$ and IL-6) also significantly decreased following treatment with CAPE in LPS-exposed cells compared with control group. Moreover, APC similarly decreased the expression levels of IL-1 $\beta$ and IL-6 (Fig. 4G and H), suggesting that inhibition of NF- $\mathrm{BB}$ signaling can downregulate miR-200c-3p, and APC had the same effect as CAPE, which decreased the expression of miR-200c-3p, IL-1 $\beta$ and IL-6. This may represent a mechanism of action underlying the effects of APC on LPS-induced lung injury.

\section{Discussion}

In the present study, APC reduced LPS-induced lung injury by upregulating ACE2 expression levels and miR-200c-3p expression was downregulated in this process. These findings 
provide insight into the underlying mechanisms of APC in the treatment of ALI/ARDS, which could present a potential therapeutic approach for severe pneumonia caused by bacterial LPS or influenza virus.

The protein encoded by the ACE2 gene belongs to the ACE family of dipeptidyl carboxydipeptidases and presents homology with ACE1 (26). The function of ACE2 is to hydrolyze angiotensin I to angiotensin (1-9) or angiotensin II to angiotensin (1-7). Angiotensin II is a pro-inflammatory molecule that directly binds to molecules of the $\mathrm{NF}-\kappa \mathrm{B}$ pathway and promotes the expression of inflammatory cytokines (27). Angiotensin (1-7) specifically binds with the Mas receptor to regulate the PIP3/AKT and ERK signaling pathways and therefore, exert anti-inflammatory effects $(28,29)$. In the lung injury model induced by ACE/angiotensin II, with the activation of the NF- $\mathrm{B}$ pathway, the expression levels of intercellular cell adhesion molecule-1 increased, resulting in increased vascular permeability and an increased degree of pulmonary edema $(27,30)$. The combination of coronavirus and ACE2 leads to a decrease in the number of available ACE2 molecules, which inhibits the conversion of angiotensin II to angiotensin (1-7). the angiotensin II produced by angiotensin I through ACE continues to increase, resulting in the accumulation of angiotensin II and aggravating inflammation (31). In the present study, in acute lung injury models exposed to LPS, the levels of ACE2 decreased and inflammatory factor levels increased, with these effects being inhibited by APC.

ACE2 has been shown to prevent acute lung injury caused by coronavirus, sepsis and influenza virus (32-34). Moreover, these studies demonstrated that avian influenza virus and bacterial LPS use a similar pathway to reduce ACE2 expression levels $(21,35)$. miRNAs can regulate gene expression by binding to the 3'-UTR of target genes (36). In addition, miRNA dysfunction has been implicated in several diseases, such as lung diseases, heart disease and diabetes (37-40). In the present study, the reported top 20 miRNAs in H5N1 virus-infected A549 cells were compared to the top 20 miRNAs targeting ACE2 and only one miRNA (miR-200c-3p) was identified that met these two conditions. In addition, following transfection with miR-200c-3p mimics, the expression levels of ACE2 were significantly downregulated in A549 cells. Notably, APC induced the downregulation of miR-200c-3p which was increased following LPS-induced injury to A549 cells. Moreover, ACE2 expression levels were also increased in the APC group. These results are consistent with previous studies $(17,22,23)$, suggesting that overexpression of ACE2 protects against LPS-induced lung injury in rats. As such, the present data showed that miR-200c-3p downregulated the expression of ACE2, and APC upregulated ACE2 expression by reducing the expression levels of $\mathrm{miR}-200 \mathrm{c}-3 \mathrm{p}$, leading to protective effects against LPS-induced lung injury.

$\mathrm{NF}-\kappa \mathrm{B}$ is a critical regulator of inflammation in ALI. Bacteria and viruses can induce activation of $N F-\kappa B$, thereby promoting inflammation (41). Previous studies have suggested that LPS activates NF- $\kappa \mathrm{B}$ through the activation of Toll-like receptors (41). In LPS-induced lung injury models and during the process of ARDS, ACE2 mitigates lung injury, likely by inhibiting NF- $\mathrm{B}$ activation (17). In the present study, APC reduced the levels of miR-200c-3p and upregulated the expression of ACE2. Furthermore, the levels of miR-200c-3p were upregulated following LPS treatment, which was suppressed by CAPE, an inhibitor of NF- $\mathrm{NB}$ (5). In addition, APC has the same inhibitory effects on $\mathrm{NF}-\kappa \mathrm{B}$ as CAPE. It has been reported that hypercapnia has a protective effect on the inhibition of key transcriptional activators in ALI inflammation through the $\mathrm{NF}-\kappa \mathrm{B}$ pathway $(3,5)$ and the present study was consistent with these findings. As such, the present results may provide a possible mechanism of action underlying the inhibition of ALI by APC.

The present study has its limitations. Firstly, virus-infected cell models were not used in this study. Secondly, it has been suggested that the LPS-induced ALI model cannot fully recapitulate the pathology observed in patients with ALI/ARDS $(42,43)$. Therefore, more clinical experiments are needed to further clarify the role of APC. In addition, in clinical practice, not all patients with ALI/ARDS require ventilator support.

In conclusion, the present study demonstrated that LPS could inhibit the expression of ACE2 by upregulating the levels of miR-200c-3p and by suppressing the NF- $\kappa$ B pathway. APC inhibited this effect, thus reducing the inflammation in LPS-induced ALI. For patients with ALI who need ventilator support, APC may represent a novel therapeutic approach with potential beneficial effects in clinical practice.

\section{Acknowledgements}

The authors would like to thank Dr Duan Shun and Dr Lan Xuekai (School of Medicine, Virginia Commonwealth University, Richmond, Virginia, USA) for their writing assistance.

\section{Funding}

This study was supported by the Foundation of Science and Technology of Jiangxi Province Plan (grant nos. 20202BABL206071 and 20181BBG78022).

\section{Availability of data and materials}

All data generated or analyzed during this study are included in this published article.

\section{Authors' contributions}

This study conception and design were performed by LQ. Acquisition of data was performed by GY, YJ, JJH, MDF and YCH. JZH performed the histological examination of the lung tissues. GY and LQ confirm the authenticity of all the raw data. Critical revision of the manuscript was given by all authors. All authors read and approved the final manuscript.

\section{Ethics approval and consent to participate}

Not applicable.

\section{Patient consent for publication}

Not applicable. 


\section{Competing interests}

The authors declare that they have no competing interests.

\section{References}

1. Ijland MM,Heunks LM and van der Hoeven JG: Bench-to-bedside review: Hypercapnic acidosis in lung injury-from 'permissive' to 'therapeutic'. Crit Care 14: 237, 2010.

2. Tan J, Liu Y, Jiang T, Wang L, Zhao C, Shen D and Cui X: Effects of hypercapnia on acute cellular rejection after lung transplantation in rats. Anesthesiology 128: 130-139, 2018.

3. Shigemura M, Lecuona E and Sznajder JI: Effects of hypercapnia on the lung. J Physiol 595: 2431-2437, 2017.

4. Laffey JG and Kavanagh BP: Hypocapnia. N Engl J Med 347: 43-53, 2002.

5. Contreras M, Masterson C and Laffey JG: Permissive hypercapnia: What to remember. Curr Opin Anesthesiol 28: 26-37, 2015.

6. Qu LC, Jiao Y, Jiang ZJ, Song ZP and Peng QH: Acidic preconditioning protects against ischemia-reperfusion lung injury via inhibiting the expression of matrix metalloproteinase 9. J Surg Res 235: 569-577, 2019.

7. Crackower MA, Sarao R, Oudit GY, Yagil C, Kozieradzki I, Scanga SE, Oliveira-dos-Santos AJ, da Costa J, Zhang L, Pei Y, et al: Angiotensin-converting enzyme 2 is an essential regulator of heart function. Nature 417: 822-828, 2002.

8. Huentelman MJ, Grobe JL, Vazquez J, Stewart JM, Mecca AP, Katovich MJ, Ferrario CM and Raizada MK: Protection from angiotensin II-induced cardiac hypertrophy and fibrosis by systemic lentiviral delivery of ACE2 in rats. Exp Physiol 90 783-790, 2005

9. Abdul-Hafez A, Mohamed T, Omar H, Shemis M and Uhal BD: The renin angiotensin system in liver and lung: Impact and therapeutic potential in organ fibrosis. J Lung Pulm Respir Res 5: 00160, 2018.

10. Oudit GY and Penninger JM: Recombinant human angiotensin-converting enzyme 2 as a new renin-angiotensin system peptidase for heart failure therapy. Curr Heart Fail Rep 8 176-183, 2011

11. Chappell MC, Marshall AC, Alzayadneh EM, Shaltout HA and Diz DI: Update on the Angiotensin converting enzyme 2-Angiotensin (1-7)-MAS receptor axis: Fetal programing, sex differences, and intracellular pathways. Front Endocrinol (Lausanne) 4: 201, 2014.

12. Kuba K, Imai Y, Ohto-Nakanishi T and Penninger JM: Trilogy of ACE2: A peptidase in the renin-angiotensin system, a SARS receptor, and a partner for amino acid transporters. Pharmacol Ther 128: 119-128, 2010.

13. Cheng H, Wang Y and Wang GQ: Organ-protective effect of angiotensin-converting enzyme 2 and its effect on the prognosis of COVID-19. J Med Virol 92: 726-730, 2020.

14. Contini C, Di Nuzzo M, Barp N, Bonazza A, De Giorgio R, Tognon $\mathrm{M}$ and Rubino S: The novel zoonotic COVID-19 pandemic: An expected global health concern. J Infect Dev Ctries 14: 254-264, 2020.

15. Danser AJ, Epstein M and Batlle D: Renin-angiotensin system blockers and the COVID-19 pandemic: At present there is no evidence to abandon renin-angiotensin system blockers Hypertension 175: 1382-1385, 2020.

16. Chen Q, Liu J, Wang W, Liu S, Yang X, Chen M, Cheng L, Lu J, Guo T and Huang F: Sini decoction ameliorates sepsis-induced acute lung injury via regulating ACE2-Ang (1-7)-Mas axis and inhibiting the MAPK signaling pathway. Biomed Pharmacother 115: 108971, 2019.

17. Li Y, Zeng Z, Cao Y, Liu Y, Ping F, Liang M, Xue Y, Xi C, Zhou M and Jiang W: Angiotensin-converting enzyme 2 prevents lipopolysaccharide-induced rat acute lung injury via suppressing the ERK1/2 and NF-кB signaling pathways. Sci Rep 6: 27911, 2016.

18. Liu Q, Du J, Yu X, Xu J, Huang F, Li X, Zhang C, Li X, Chang J, Shang D, et al: miRNA-200c-3p is crucial in acute respiratory distress syndrome. Cell Discov 3: 17021, 2017.

19. Zhang X, Huang H, Yang T, Ye Y, Shan J, Yin Z and Luo L: Chlorogenic acid protects mice against lipopolysaccharide-induced acute lung injury. Injury 41: 746-752, 2010.

20. Zhang CH, Fan YY, Wang XF, Xiong JY, Tang YY, Gao JQ, Shen Z, Song XH, Zhang JY, Shen Y, et al: Acidic preconditioning protects against ischemia-induced brain injury. Neurosci Lett 523: 3-8, 2012.
21. Gu H, Xie Z, Li T, Zhang S, Lai C, Zhu P, Wang K, Han L, Duan Y, Zhao Z, et al: Angiotensin-converting enzyme 2 inhibits lung injury induced by respiratory syncytial virus. Sci Rep 6: 19840, 2016.

22. Livak KJ and Schmittgen TD: Analysis of relative gene expression data using real-time quantitative PCR and the 2(-Delta Delta C(T)) method. Methods 25: 402-408, 2001.

23. Ye R and Liu Z: ACE2 exhibits protective effects against LPS-induced acute lung injury in mice by inhibiting the LPS-TLR4 pathway. Exp Mol Pathol 113: 104350, 2020.

24. Niu J, Shi Y, Tan G, Yang CH, Fan M, Pfeffer LM and Wu ZH: DNA damage induces NF- $\kappa$ B-dependent microRNA-21 up-regulation and promotes breast cancer cell invasion. J Biol Chem 287: 21783-21795, 2012.

25. Zhou R, Hu G, Liu J, Gong AY, Drescher KM and Chen XM: NF-kappaB p65-dependent transactivation of miRNA genes following Cryptosporidium parvum infection stimulates epithelial cell immune responses. PLoS Pathog 5: e1000681, 2009.

26. Minato T, Nirasawa S, Sato T, Yamaguchi T, Hoshizaki M, Inagaki T, Nakahara K, Yoshihashi T, Ozawa R, Yokota S, et al: B38-CAP is a bacteria-derived ACE2-like enzyme that suppresses hypertension and cardiac dysfunction. Nat Commun 11: 1058, 2020.

27. Celec P: Nuclear factor kappa B-molecular biomedicine: The next generation. Biomed Pharmacother 58: 365-371, 2004.

28. Kvandova $\mathrm{M}$ and Dovinova I: Functioning of the PPAR gamma and its effect on cardiovascular and metabolic Diseases. In: Metabolic Syndrome, edition: 160 Greentree Drive, Suite 101, Dover, DE 19904, USA, pp1-41, 2017 http://smgebooks. com/metabolic-syndrome/index.php.

29. Passos-SilvaDG, Verano-BragaT and Santos RA: Angiotensin-(1-7): Beyond the cardio-renal actions. Clin Sci 124: 443-456, 2013.

30. Rodrigues Prestes TR, Rocha NP, Miranda AS, Teixeira AL and Simoes-E-Silva AC: The anti-inflammatory potential of ACE2/angiotensin-(1-7)/mas receptor axis: evidence from basic and clinical research. Current Drug Targets 18: 1301-1313, 2017.

31. Kuba K, Imai Y, Rao S, Jiang C and Penninger JM: Lessons from SARS: Control of acute lung failure by the SARS receptor ACE2. J Mol Med 84: 814-820, 2006.

32. Zou Z, Yan Y, Shu Y, Gao R, Sun Y, Li X, Ju X, Liang Z, Liu Q, Zhao $\mathrm{Y}$, et al: Angiotensin-converting enzyme 2 protects from lethal avian influenza A H5N1 infections. Nat Commun 5: 3594, 2014.

33. Wang J, Zhao S, Liu M, Zhao Z, Xu Y, Wang P, Lin M, Xu Y, Huang B, Zuo X, et al: ACE2 expression by colonic epithelial cells is associated with viral infection, immunity and energy metabolism. medRxiv, Feb 7, 2020 doi: https://doi.org/10.1101/ 2020.02.05.20020545

34. Gralinski LE, Sheahan TP, Morrison TE, Menachery VD, Jensen K, Leist SR, Whitmore A, Heise MT and Baric RS: Complement activation contributes to severe acute respiratory syndrome coronavirus pathogenesis. MBio 9: e01753-18, 2018.

35. Imai Y, Kuba K, Rao S, Huan Y, Guo F, Guan B, Yang P, Sarao R, Wada T, Leong-Poi H, et al: Angiotensin-converting enzyme 2 protects from severe acute lung failure. Nature 436: 112-116, 2005.

36. Ebert MS and Sharp PA: Emerging roles for natural microRNA sponges. Curr Biol 20: R858-R861, 2010.

37. Christopher AF, Kaur RP, Kaur G, Kaur A, Gupta V and Bansal P: MicroRNA therapeutics: Discovering novel targets and developing specific therapy. Perspect Clin Res 7: 68-74, 2016.

38. Magenta A, Greco S, Gaetano C and Martelli F: Oxidative stress and microRNAs in vascular diseases. Int J Mol Sci 14: 17319-17346, 2013.

39. Zhang HN, Xu QQ, Thakur A, Alfred MO, Chakraborty M, Ghosh A and Yu XB: Endothelial dysfunction in diabetes and hypertension: Role of microRNAs and long non-coding RNAs. Life Sci 213: 258-268, 2018.

40. Caballero AE: Endothelial dysfunction in obesity and insulin resistance: A road to diabetes and heart disease. Obes Res 11: 1278-1289, 2003

41. Alexopoulou L, Holt AC, Medzhitov R and Flavell RA Recognition of double-stranded RNA and activation of NF- $\kappa \mathrm{B}$ by Toll-like receptor 3. Nature 413: 732-738, 2001.

42. Uchida T, Shirasawa M, Ware LB, Kojima K, Hata Y, Makita K, Mednick G, Matthay ZA and Matthay MA: Receptor for advanced glycation end-products is a marker of type I cell injury in acute lung injury. Am J Respir Criti Care Med 173: 1008-1015, 2006.

43. Proudfoot AG, McAuley DF, Griffiths MJ and Hind M: Human models of acute lung injury. Dis Models Mech 4: 145-153, 2011.

This work is licensed under a Creative Commons Attribution-NonCommercial-NoDerivatives 4.0 International (CC BY-NC-ND 4.0) License. 